\title{
The Hard State of the Highly Absorbed High Inclination Black Hole Binary Candidate Swift J1658.2-4242 Observed by NuSTAR and Swift
}

\author{
Yanjun $\mathrm{Xu}^{1}$ (10), Fiona A. Harrison ${ }^{1}$ (1D), Jamie A. Kennea ${ }^{2}$ (D), Dominic J. Walton ${ }^{3}$ (D), John A. Tomsick ${ }^{4}$ (D), Jon M. Miller ${ }^{5}$, \\ Didier Barret $^{6,7}$ (D), Andrew C. Fabian ${ }^{3}$ (D), Karl Forster ${ }^{1}$, Felix Fürst ${ }^{8}$ (D), Poshak Gandhi ${ }^{9}$ (D), and Javier A. García ${ }^{1,10}$ (D) \\ ${ }^{1}$ Cahill Center for Astronomy and Astrophysics, California Institute of Technology, Pasadena, CA 91125, USA \\ ${ }^{2}$ Department of Astronomy and Astrophysics, Pennsylvania State University, 525 Davey Lab, University Park, PA 16802, USA \\ ${ }^{3}$ Institute of Astronomy, University of Cambridge, Madingley Road, Cambridge CB3 0HA, UK \\ ${ }^{4}$ Space Sciences Laboratory, 7 Gauss Way, University of California, Berkeley, CA 94720-7450, USA \\ ${ }^{5}$ Department of Astronomy, University of Michigan, 1085 South University Avenue, Ann Arbor, MI 48109, USA \\ ${ }^{6}$ Universite de Toulouse, UPS-OMP, IRAP, Toulouse, France \\ ${ }^{7}$ CNRS, IRAP, 9 Av. colonel Roche, BP 44346, F-31028 Toulouse cedex 4, France \\ ${ }^{8}$ European Space Astronomy Centre (ESA/ESAC), Operations Department, Villanueva de la Cañada (Madrid), Spain \\ ${ }^{9}$ Department of Physics and Astronomy, University of Southampton, SO17 3RT, UK \\ ${ }^{10}$ Remeis Observatory \& ECAP, Universität Erlangen-Nürnberg, Sternwartstr. 7, D-96049 Bamberg, Germany \\ Received 2018 May 18; revised 2018 July 30; accepted 2018 August 9; published 2018 September 18
}

\begin{abstract}
We present a spectral and timing analysis of the newly reported Galactic X-ray transient Swift J1658.2-4242 observed by Nuclear Spectroscopic Telescope Array (NuSTAR) and Swift. The broadband X-ray continuum is typical of a black hole binary in the bright hard state, with a photon index of $\Gamma=1.63 \pm 0.02$ and a low coronal temperature of $k T_{\mathrm{e}}=22 \pm 1 \mathrm{keV}$, corresponding to a low spectral cutoff well constrained by NuSTAR. Spectral modeling of the relativistic disk reflection features, consisting of a broad Fe $\mathrm{K} \alpha$ line and the Compton reflection hump, reveals that the black hole is rapidly spinning with the spin parameter of $a^{*}>0.96$, and the inner accretion disk is viewed at a high inclination angle of $i=64_{-3}^{+2 \circ}$ (statistical errors, 90\% confidence). The high inclination is independently confirmed by dips in the light curves, which can be explained by absorbing material located near the disk plane temporarily obscuring the central region. In addition, we detect an absorption line in the NuSTAR spectra centered at $7.03_{-0.03}^{+0.04} \mathrm{keV}$. If associated with ionized $\mathrm{Fe} \mathrm{K}$ absorption lines, this provides evidence for the presence of outflowing material in the low/hard state of a black hole binary candidate. A timing analysis shows the presence of a type-C quasi-periodic oscillation in the power spectrum, with the frequency increasing from $\sim 0.14$ to $\sim 0.21 \mathrm{~Hz}$ during the single NUSTAR exposure. Our analysis reveals that Swift J1658.2-4242 displays characteristics typical for a black hole binary that is viewed at a high inclination angle, making it a good system for studying the accretion geometry in black hole binaries.
\end{abstract}

Key words: accretion, accretion disks - X-rays: binaries - X-rays: individual (Swift J1658.2-4242)

\section{Introduction}

Most Galactic black hole binaries are discovered as X-ray transients that go into recurrent outbursts. During a typical outburst, a black hole binary transitions from the low/hard state (power-law component dominates the energy spectrum with a hard photon index) to the high/soft state (thermal disk component dominates) as the source flux increases (see Remillard \& McClintock 2006 for a review). The changes in the spectral shape are believed to be associated with the evolution of the accretion geometry. One hypothesis that has been widely explored is that the inner disk extends to the innermost stable circular orbit (ISCO) in the soft state, whereas in the hard state the inner disk is truncated at a large radius (e.g., Esin et al. 1997; Done et al. 2007). However, it has been suggested from observations that the inner disk extends down to the ISCO in the hard state of black hole binaries in several cases (e.g., Miller et al. 2002, 2006). Whether or not the accretion disk is truncated can be determined by measuring the degree of relativistic distortion of the $\mathrm{Fe} \mathrm{K} \alpha$ emission line, which comes from reflection of the central emission by the inner accretion disk (e.g., Fabian et al. 1989). Recently, NUSTAR observations of several black hole binaries in the bright hard state revealed very broad $\mathrm{Fe} \mathrm{K} \alpha$ lines (Miller et al. 2015b; El-Batal et al. 2016; Xu et al. 2018b). As NuSTAR spectra are free from pile-up distortions even at high count rates typical of Galactic X-ray binaries, these results clearly challenge the disk truncation interpretation of the hard state.

Mass outflows are important phenomena in the study of accretion processes. Equatorial disk winds, identified by narrow absorption lines from highly ionized iron (mostly Fe XXV and Fe XXVI), have been observed in a number of black hole binaries in the soft state, and are more likely to be observed in high inclination systems (e.g., Neilsen \& Lee 2009; King et al. 2014; Miller et al. 2015a). Detailed studies of several well-known black hole binaries indicate that the presence of accretion disk winds appears to be anticorrelated with relativistic jets (e.g., Neilsen \& Lee 2009; Miller et al. 2012). The disk wind is thought to be responsible for carrying away a considerable amount of kinetic energy when the jet switches off in the high/soft state. During typical low/ hard states when the source Eddington ratio is relatively low, however, collimated radio jets are ubiquitous, and disk wind features are either absent or at least much weaker, thus making detection difficult (e.g., Miller et al. 2012; Ponti et al. 2012).

Swift J1658.2-4242 is a newly discovered X-ray transient in the Galactic plane. The first reported detection was made by Swift/BAT (Krimm et al. 2013) on 2018 February 16. The source was detected by INTEGRAL during its observations of the Galactic center field on 2018 February 13, with a spectral 
hardness typical for black hole binaries in the hard state (Grebenev et al. 2018; Grinberg et al. 2018). Subsequent radio observations by ATCA imply that the source is a black hole binary at a distance of greater than $3 \mathrm{kpc}$ (Russell et al. 2018). Low-frequency quasi-periodic oscillations (QPOs) increasing in frequency were detected by $\operatorname{NuSTAR}$ (Xu et al. 2018a) and AstroSAT (Beri et al. 2018). X-ray spectra from Swift/XRT reveal that the source is highly absorbed, with an absorption column density of $N_{\mathrm{H}}>10^{23} \mathrm{~cm}^{-2}$ (Lien et al. 2018). In addition, several dips were observed from the initial analysis of the NUSTAR data (Xu et al. 2018a), similar to those detected in known dipping low-mass X-ray binaries (e.g., Tomsick et al. 1998; Díaz Trigo et al. 2009; Kuulkers et al. 2013). Dips in the light curves of black hole and neutron star binaries are signatures of high inclination $\left(i>65^{\circ}\right)$. They are thought to originate from obscuring material located at the thick outer region of the accretion disk in low-mass X-ray binaries (White \& Swank 1982; Frank et al. 1987), while in high-mass X-ray binaries, dips can be explained by obscuration by the stellar wind from the donor star.

In this paper, we present a spectral and timing analysis of the newly discovered black hole binary candidate Swift J1658.2-4242, using simultaneous NuSTAR and Swift observations that caught the source in the hard state. The paper is structured as follows: in Section 2, we describe the observations and the data reduction details; we present the results of our spectral modeling and timing analysis in Sections 3, 4, and 5, and discuss the results in Section 6.

\section{Observations and Data Reduction}

Swift J1658.2-4242 was observed by NuSTAR (Harrison et al. 2013) on 2018 February 16 starting at 23:26:09 UT for an exposure of $33.3 \mathrm{ks}$ per module (OBSID: 90401307002). The observation was taken on the same day that the outburst was first reported. We reduced the NUSTAR data following standard procedure using NuSTARDAS pipeline v.1.6.0 and CALDB v20170817. The source spectra were extracted from a circular region with the radius of $150^{\prime \prime}$ from the two NUSTAR focal plane modules (FPMA and FPMB). Corresponding background spectra were extracted using polygonal regions from source-free areas in the detectors. We also extracted spectra from mode 6 data following the procedures described in Walton et al. (2016), which yielded an extra $\sim 3.6 \mathrm{ks}$ of data when an aspect solution was not available from the on board star tracker CHU4. We grouped the NuSTAR spectra to achieve a signal-to-noise ratio $(\mathrm{S} / \mathrm{N})$ of 20 per energy bin.

The outburst of Swift J1658.2-4242 was monitored by Swift/XRT (Burrows et al. 2005). The XRT observation (OBS ID: 00810300002) overlapping with the NuSTAR observation used in this paper was taken in the windowed timing (WT) mode to avoid photon pile-up. The XRT observation was taken around the middle of the NuSTAR exposure, starting from 10:38:52 to 16:43:57 UT on 2018 February 17. We reduced the the Swift/XRT data using xrtpipeline v.0.13.2 with CALDB v20171113. We extracted the source spectrum from a circular region with the radius of $70^{\prime \prime}$, and the background was extracted from an annulus area with the inner and outer radii of $200^{\prime \prime}$ and $300^{\prime \prime}$, respectively. After standard data filtering, the total exposure time is $3.9 \mathrm{ks}$. The XRT spectrum was rebinned to have at an $\mathrm{S} / \mathrm{N}$ of at least 5 per bin.

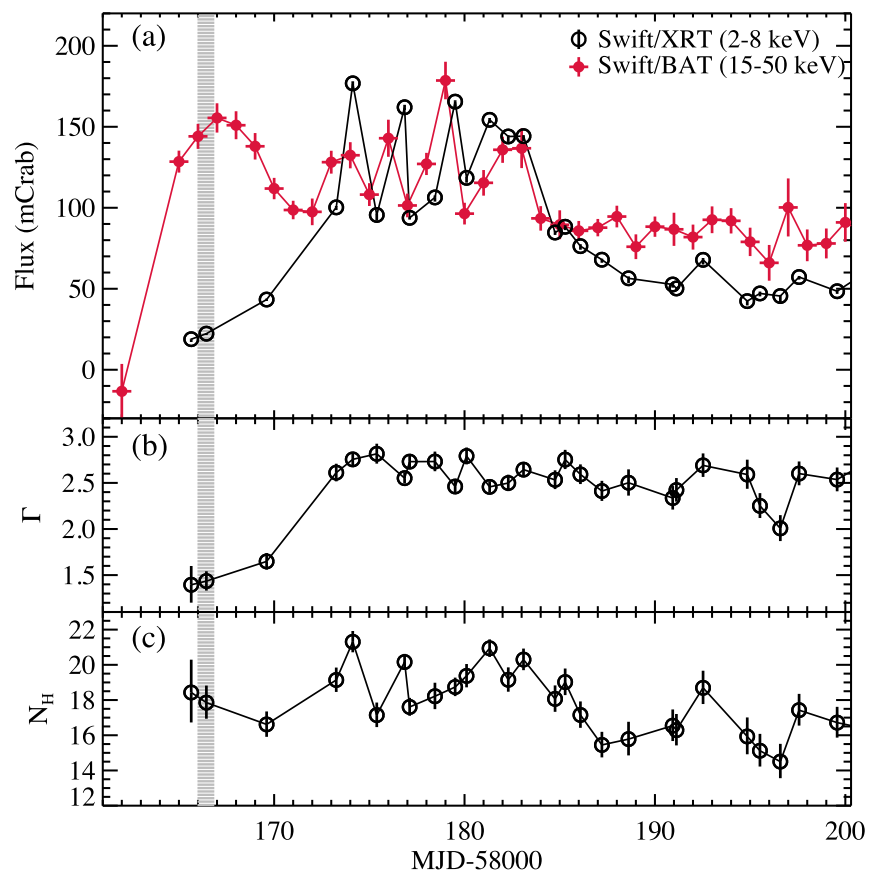

Figure 1. (a) Swift monitoring of the outburst of Swift J1658.2-4242. The BAT light curve is rescaled to the unit mCrab from count rates in the $15-50 \mathrm{keV}$ band. The XRT light curve is converted to mCrab using calculated flux in the $2-8 \mathrm{keV}$ band. (b), (c) Photon index $\Gamma$ and absorption column density $N_{\mathrm{H}}$ derived by fitting the XRT spectra with an absorbed power-law model. Gray shaded area marks the duration of the NuSTAR observation.

\section{Light Curves}

Evolution of the flux of Swift J1658.2-4242 since the beginning of the outburst observed by Swift/XRT and BAT is shown in Figure 1. The Swift/BAT light curve in daily averaged flux is from the Swift/BAT transient monitor (Krimm et al. 2013). Swift/XRT data were reduced following the procedures described in Section 2, and the XRT flux was calculated by fitting the spectra with an absorbed power-law model, TBabs*powerlaw. In this work, we perform all spectral fitting using XSPEC v12.9.0n (Arnaud 1996) with $\chi^{2}$ statistics, and adopt the cross-sections from Verner et al. (1996) and abundances from Wilms et al. (2000). All parameter uncertainties are reported at the $90 \%$ confidence level for one parameter of interest unless otherwise clarified. As shown in Figure 1(b), the NuSTAR observation caught Swift J1658.2-4242 in the hard state before significant spectral softening occurred.

Three dips are evident in the NuSTAR light curve generated by the standard NUPRODUCTS procedure, where the NuSTAR full band count rate decreased by $\sim 45 \%-70 \%$ at the dip minima (see Figure 2(a)). Dipping has been found to be periodic in the black hole binaries GRO J1655-40 and MAXI J1659-152, and is associated with the binary orbital period in these systems (Kuulkers et al. 1998, 2013). It is possible that the dips observed in Swift J1658.2-4242 are also periodic, but cannot be confirmed with the limited exposure time of our NUSTAR observation. We extract source light curves from three energy intervals, $3-6 \mathrm{keV}(S), 6-10 \mathrm{keV}(M)$, and $10-79 \mathrm{keV}(H)$, and calculate the hardness ratios defined as $\operatorname{HR} 1=(M-S)$ / $(M+S)$ and HR2 $=(H-M) /(H+M)$. The dips are clearly detected in all three energy bands. The hardness ratios are found 


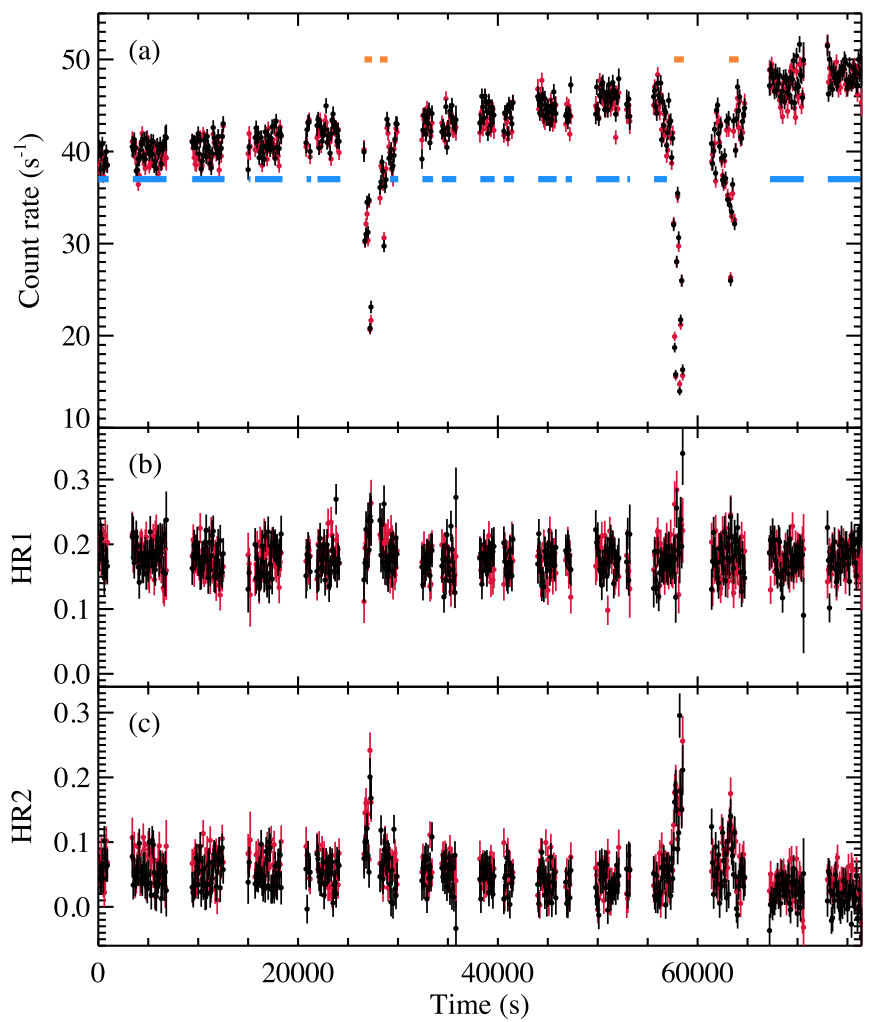

Figure 2. (a) NUSTAR full band light curves of Swift J1658.2-4242 in $100 \mathrm{~s}$ bins (FPMA in black, FPMB in red). Orange and blue lines mark the data intervals selected for the dip and persistent spectra, respectively. The orbital gaps in the light curves are due to occultations and South Atlantic Anomaly (SAA) passages. (b), (c) Plots of corresponding hardness ratios. Hardness ratios HR1 and HR2 are defined as HR1 $=(M-S) /(M+S)$ and HR2 $=(H-M) /$ $(H+M)$, where $S, M$, and $H$ are the count rates in the energy bands of 3-6 keV, 6-10 keV, and 10-79 keV, respectively.

to increase during the dips (see Figures 2(b), (c)), indicating the dips are caused by increased absorption along the line of sight.

The source count rate was rising linearly during the $N u S T A R$ observation. Despite the increase in count rate, the hardness ratio, HR1, basically remains constant outside of the dips. The overall change in HR2 is also relatively small (decreased by $\sim 0.03$ by the end of the exposure), which in this case is mainly due to the change in the high-energy cutoff. The cutoff energy, $E_{\text {cut }}$, in black hole binaries has been observed to decrease monotonically with the increasing flux during the rising phases of their hard states (e.g., Joinet et al. 2008; Motta et al. 2009). We note here that the corresponding changes in the absolute values of spectral parameters are subtle. Therefore, for spectral modeling, we only consider time-averaged spectra and separate the total exposure by dipping and non-dipping phases.

\section{Spectral Analysis}

\subsection{Persistent Spectra}

We first consider time-averaged spectra excluding the dips, which we refer to as the persistent spectra henceforth. Although the exposure of Swift/XRT is much shorter than that of the NuSTAR observation, Swift caught the second dip observed by NuSTAR, which we also excluded from the XRT data when extracting the persistent spectrum. The accumulated times for the persistent spectra are $30.9 \mathrm{ks}, 31.4 \mathrm{ks}$, and $3.6 \mathrm{ks}$ for NuSTAR/ FPMA, NuSTAR/FPMB, and Swift/XRT, respectively.

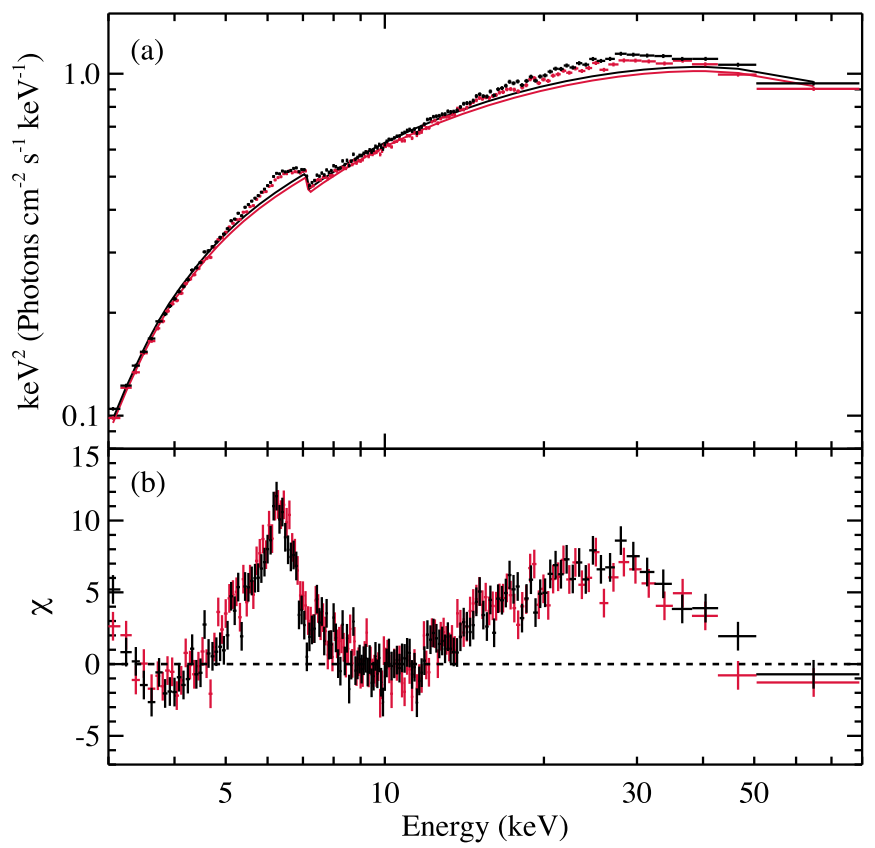

Figure 3. (a) Persistent (non-dip) hard state spectra of Swift J1658.2-4242 observed by NUSTAR. FPMA and FPMB data are plotted in black and red, respectively. The energy spectra are folded with a cutoff power-law model. (b) Clear relativistic reflection features can be seen in the spectral residuals plotted in $\chi$. The spectra have been rebinned for display clarity.

Relativistic reflection features are clearly detected in the NUSTAR spectra, including a broad $\mathrm{Fe} \mathrm{K} \alpha$ line and a Compton reflection hump peaking around $30 \mathrm{keV}$ (see Figure 3). To highlight the disk reflection features, we first fit the NUSTAR spectra with an absorbed cutoff power-law model, TBnew*cutoffpl, in XSPEC notation, only considering the energy intervals of 3-5, 8-12, and 40-79 keV. We note that in the residual plot, there is some indication for an absorption line around $7 \mathrm{keV}$ near the absorption edge (see Figure 3(b)). Throughout this work, we use the TBnew ${ }^{11}$ model to account for neutral absorption, and fix all abundances at solar in TBnew. This updated version of the TBabs model better characterizes the shape of the absorption edges, which could be relevant here as the source has a high absorption column density. This approximate fit requires an absorption column density of $N_{\mathrm{H}} \sim 1.3 \times 10^{23} \mathrm{~cm}^{-2}$, a photon index of $\Gamma \sim 1.3$, and a relatively low cutoff energy of $E_{\text {cut }} \sim 55 \mathrm{keV}$.

To obtain broadband X-ray coverage so that spectral parameters can be better constrained, we jointly fit the NUSTAR and the Swift/XRT spectra. We allow the cross-normalization constants to vary freely for $N u S T A R / F P M B$ and Swift/XRT, and fix the value at unity for $N u S T A R / F P M A$. We use XRT data above $1 \mathrm{keV}$, as low-energy spectral residuals have been reported in heavily absorbed sources observed in the WT mode. ${ }^{12}$ Also NuSTAR data below $4 \mathrm{keV}$ are ignored during the spectral fitting to avoid possible calibration uncertainties near the low-energy end of the band pass. We use the model relxilllpcp in the relxill model family (Dauser et al. 2014; García et al. 2014) to physically model the relativistic reflection spectra. The relxilllpCp model uses an idealized lamppost geometry for the illuminating source commonly referred to as the corona. In this model, the corona

\footnotetext{
11 http://pulsar.sternwarte.uni-erlangen.de/wilms/research/tbabs/

12 http://www.swift.ac.uk/analysis/xrt/digest_cal.php
} 
is approximated as a point source located on the spin axis of the black hole above the accretion disk. The reflection fraction, $R_{\text {ref, }}$ can be self-consistently calculated in the lamppost model based on the geometry assumed, which helps to reduce the parameter space. It uses the thermal Comptonization model nthcomp (Zdziarski et al. 1996; Życki et al. 1999) as the input continuum, which provides a generally different spectral curvature from the phenomenological cutoffpl model, especially in the case of a low high-energy cutoff. For all spectral fittings with relxilllpcp, we fix the outer radius of the accretion disk, $R_{\text {out }}$, at $400 r_{\mathrm{g}}\left(r_{\mathrm{g}} \equiv \mathrm{GM} / c^{2}\right.$ is the gravitational radius), and set the value of reflection fraction, $R_{\text {ref, }}$, to be self-consistently determined by the model given the combination of the black hole spin parameter $a^{*}$, the inner radius of the accretion disk $R_{\text {in }}$, and the lamppost height $h$. The inner disk radius, $R_{\mathrm{in}}$, and the black hole spin parameter, $a^{*}$, are degenerate, as they both control the effective inner accretion disk radius. Therefore, for simplicity, during the spectral fitting we assume that the inner disk extends down to the ISCO by fixing $R_{\text {in }}$ at the radius of the ISCO, and fit for the black hole spin as a free parameter (see Section 6 for a discussion about disk truncation).

It is clear that disk reflection alone, TBabs*relxilllpCp (Model 1), is not sufficient to describe the data (reduced chisquared $\chi^{2} / \nu=1808.8 / 1599=1.13$, where $\nu$ is the number of degrees of freedom). Secondary features including a narrow iron line and possible absorption around $7 \mathrm{keV}$ can be seen in the residual plot (see Figure 4(b)). A narrow iron line component on top of the broad $\mathrm{Fe} \mathrm{K}$ profile has been found in a number of black hole binaries observed by NuSTAR (e.g., Walton et al. 2016, 2017; Tomsick et al. 2018; Xu et al. 2018 b), although the narrow line complex cannot be spectrally resolved. The origin of this spectral component is still controversial. Possible scenarios generally considered include distant reflection by a flared disk, line emission from the stellar wind of the donor star, or re-emission from an accretion disk wind if detected along with absorption features. We first tentatively fit for this feature using an unblurred reflection model, xillverCp (García \& Kallman 2010), to account for possible contribution from distant reflection, with the total model set up as TBabs*(relxilllpCp+xillverCp) (Model 2). We assume the narrow iron line to be neutral by setting the ionization parameter in xillverCp as $\log \xi=0$ ( $\xi=4 \pi F_{\mathrm{x}} / n$, where $F_{\mathrm{x}}$ is the ionizing flux and $n$ is the gas density). We link other parameters in xillvercp with the corresponding parameters in relxilllpcp, only allowing the normalization of $\mathrm{xillverCp}$ to vary freely. This results in an improvement of $\Delta \chi^{2}=93$ with one additional free parameter $\left(\chi^{2} / \nu=1715.8 / 1598=1.07\right)$. Modeling the narrow iron line with relxilllpCp instead results in a similar fit, and it yields a lower limit on the distance of the distant reflection component $\left(>300 r_{\mathrm{g}}\right.$ ), which is far enough from the black hole to justify its low ionization state.

An apparent absorption line is still evident in the residuals of Model 2 (see Figure 4(c)). Fitting this feature with a simple Gaussian absorption line model brings an improvement of $\Delta \chi^{2}=67.5$ to the fit with three more parameters. It reveals that the line centroid lies at $E_{\text {abs }}=7.03_{-0.03}^{+0.04} \mathrm{keV}$, with an equivalent width of $23_{-4}^{+5} \mathrm{eV}$. The absorption line is significantly detected at $7.5 \sigma$ confidence via a simple F-test. Strong absorption features from highly ionized iron are unusual for black hole binaries in the low/hard state, as they are considered

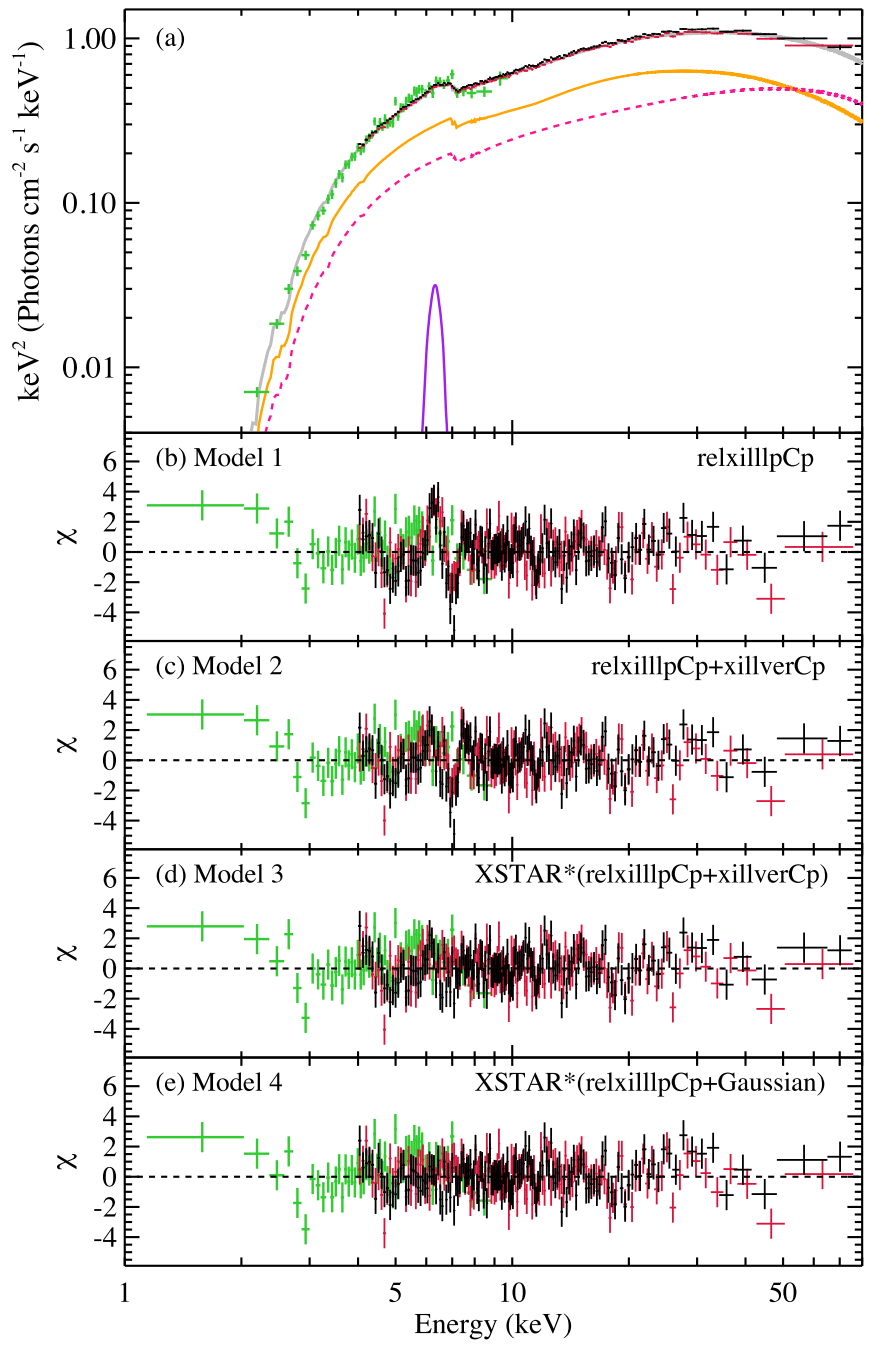

Figure 4. (a) Persistent broadband X-ray spectra of Swift J1658.2-4242 folded with the best-fit model (Model 4). The total model is marked in gray solid lines, with contributions from the Comptonization continuum (magenta), the disk reflection (orange), and the narrow Fe K line (purple). The Swift/XRT spectrum is plotted in green, and the same color scheme is used for the NuSTAR data as in previous plots. The spectra are rebinned for display clarity. (b)-(e) Spectral modeling residuals.

as signatures of a powerful disk wind. Complications arise from the fact that the apparent absorption line is right at the $\mathrm{Fe}$ K-edge. Assuming this is indeed an iron absorption line originating from a disk wind, if associated with blueshifted Helike Fe XXV $(6.70 \mathrm{keV})$, it requires an extreme outflowing velocity of $0.049_{-0.004}^{+0.006} c\left(14800_{-1300}^{+1800} \mathrm{~km} \mathrm{~s}^{-1}\right)$; alternatively, if this is identified with the more ionized H-like Fe XXVI $(6.97 \mathrm{keV})$, it corresponds to a lower velocity of $0.009_{-0.004}^{+0.006} c$ $\left(2600_{-1300}^{+1800} \mathrm{~km} \mathrm{~s}^{-1}\right)$, but is still a high outflowing velocity for a disk wind launched by a stellar-mass black hole (e.g., Neilsen \& Lee 2009; Miller et al. 2015a). However, we note that in the latter case, the velocity shift could only be marginal when taking into account the absolute energy calibration uncertainty of NUSTAR (Madsen et al. 2015).

In order to obtain a more physical interpretation, we fit for the absorption feature with an XSTAR table model (Kallman \& Bautista 2001). The full model is set up as TBabs*XSTAR* (relxilllpcp+xillverCp) (Model 3). The XSTAR photoionization grid was customized for the hard state of Swift J1658.2-4242 using a power-law input spectrum with the 


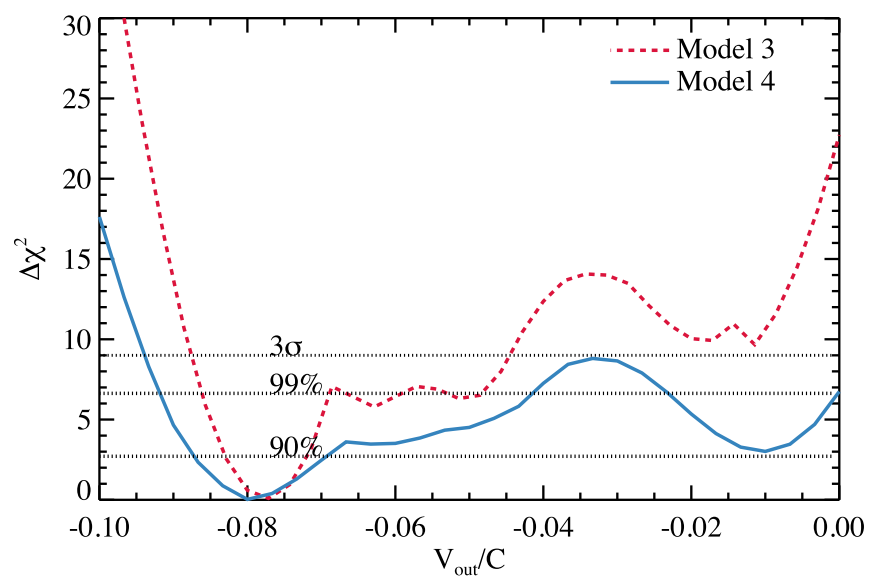

Figure 5. $\Delta \chi^{2}$ plot of the velocity shift of the ionized absorber. The dashed lines mark the $90 \%, 99 \%$, and $3 \sigma$ confidence levels for one parameter of interest.

photon index of $\Gamma=1.7$. We also assumed a gas density of $n=10^{14} \mathrm{~cm}^{-3}$ following Miller et al. (2015a), a turbulent velocity of $1000 \mathrm{~km} \mathrm{~s}^{-1}$, and a source luminosity of $L=$ $10^{38} \mathrm{erg} \mathrm{s}^{-1}$. The grid covers the parameter space of $1.5 \leqslant$ $\log (\xi) \leqslant 4.5,10^{21} \mathrm{~cm}^{-2} \leqslant N_{\mathrm{H}} \leqslant 10^{23} \mathrm{~cm}^{-2}$, and $0.1 \leqslant A_{\mathrm{Fe}} \leqslant$ 10.0 , with the velocity shift as a free parameter. We link the iron abundance of the XSTAR model (in solar units), $A_{\mathrm{Fe}}$, with that of the disk reflection component. The XSTAR model successfully accounts for the absorption feature with an ionization parameter of $\log (\xi)=2.09_{-0.07}^{+0.17}$, and a very high outflowing velocity shift of $v_{\text {out }}=0.077_{-0.007}^{+0.006} c\left(22800 \pm 1800 \mathrm{~km} \mathrm{~s}^{-1}\right)$. We stress that obtaining an unique constraint on the ionization parameter and the velocity shift simultaneously is difficult here. Since only one absorption line can been clearly seen in the Fe K band, the ionization parameter and the velocity shift of the XSTAR grid are degenerate. At $3 \sigma$ confidence level, a velocity of $v_{\text {out }}$ at $\sim 0.11 c\left(3300 \mathrm{~km} \mathrm{~s}^{-1}\right)$ can still be considered as marginally acceptable (see Figure 5 for the constraint on $v_{\text {out }}$ ). It indicates that associating the absorption feature with more highly ionized $\mathrm{Fe} \mathrm{K}$ species cannot be completely ruled out. The heavily absorbed nature of the source plus the short XRT exposure time precludes the possibility of detecting corresponding absorption lines in the soft end of the spectra. Model 3 provides an overall satisfying fit of the spectra, the results indicate that the source is a rapidly spinning black hole with the spin parameter of $a^{*}=0.92_{-0.06}^{+0.04}$ and is viewed at a high inclination angle of $i=61_{-4}^{+3 \circ}$.

In an attempt to further improve the fit, we replace the xillverCp component with a simple Gaussian emission line model (Model 4), since there is no clear indication that the narrow core of the $\mathrm{Fe} \mathrm{K} \alpha$ profile arises from reflection. This results in a better fit by $\Delta \chi^{2}=25$, with the difference mainly lying at the red wing of the narrow iron line around $6 \mathrm{keV}$ (see Figure 4). The inclusion of an extra disk blackbody component (diskbb) is not statistically required by the data. As the spectra display no obvious bump-like feature at low energies, most likely due to the high absorption column density, the strength or the temperature of the thermal disk emission cannot be well constrained. Although thermal emission from the inner accretion disk is physically required to explain a non-truncated disk, including a diskbb model or not does not significantly affect the determination of other parameters during spectral modeling. Therefore, we consider Model 4 as our best-fit model for the persistent broadband X-ray spectra of Swift J1658.2-4242.

Broadly similar results are obtained with Model 4: a low coronal height of $h<3.8 r_{\mathrm{g}}$ and a high spin of $a^{*}>0.96$, which lead to a high reflection fraction of $R_{\text {ref }}=3.25$ calculated self-consistently by the model, signaling strong light-bending effects at the vicinity of the black hole. A high inclination angle of $i=64_{-3}^{+2} \circ$ is measured for the inner accretion disk, which is sensitive to the blue wing of the broad iron line. Similar results are obtained if $R_{\text {ref }}$ is left to vary freely, and an equally high black hole spin is measured, indicating that the high reflection fraction is indeed required by the data. We note that consistent results are obtained if we replace the lamppost model used here ( $r e l \times i l l l p C p)$ with the model assuming a broken power-law coronal emissivity profile (relxillcp); the best-fit values of the key physical parameters (black hole spin, disk ionization, and inclination) are all consistent within errors. The constraint on the velocity shift of the XSTAR absorption component with Model 4 is weaker compared to that with Model 3. By allowing both line the width and centroid to vary in the Gaussian emission line model, more flexibilities are brought to the spectral modeling near the Fe K-edge. However, Model 4 still statistically prefers a considerably large outflowing velocity for the ionized absorber (see Figure 5). The most noticeable difference between Model 4 and 3 is the iron abundance, $A_{\mathrm{Fe}}$, and the photon index, $\Gamma$ (see Table 1 for the list of spectral modeling parameters). The determination of $A_{\mathrm{Fe}}$ is driven by the relative flux in the Fe $\mathrm{K}$ band and of the Compton hump. Different from the distant reflection model, the narrow iron line component in Model 4 does not contribute to the flux in the high-energy band $(>10 \mathrm{keV})$. This would cause a small change in the overall spectral shape, resulting in corresponding changes in $\Gamma$ and $A_{\mathrm{Fe}}$.

The fit confirms that the source is highly absorbed. The best-fit neutral absorption column density, $N_{\mathrm{H}}=1.81_{-0.04}^{+0.07} \times$ $10^{23} \mathrm{~cm}^{-2}$, is consistent with the measurement reported from the initial analysis of the Swift/XRT data $\left(N_{\mathrm{H}}=(1.9 \pm 0.5) \times\right.$ $10^{23} \mathrm{~cm}^{-2}$; Lien et al. 2018). This value is well in excess of the expected Galactic absorption in the direction of Swift J1658.2-4242, $N_{\mathrm{H}, \mathrm{Gal}}=1.55 \times 10^{22} \mathrm{~cm}^{-2}$ (Kalberla et al. 2005). Therefore, the extra absorption is intrinsic to the source, and most likely originates from obscuring material near the orbital plane of the system. The parameters of the nthcomp continuum are well constrained: a power-law index of $\Gamma=$ $1.63 \pm 0.01$, indicating the source is in the hard state, and a relatively low coronal temperature of $k T_{\mathrm{e}}=22 \pm 1 \mathrm{keV}$ (the value here is as observed, not corrected for gravitational redshift at the vicinity of the black hole), which is determined by the exponential cutoff at the high-energy end of the spectrum. Similar low coronal temperatures or equivalently low high-energy cutoffs have been previously measured by NUSTAR in black hole binaries during their bright hard states (e.g., Miller et al. 2013, 2015b; Xu et al. 2018b).

\subsection{Dip Spectra}

We extracted the spectra of the three dips collectively to obtain the highest $\mathrm{S} / \mathrm{N}$ data possible for spectral fitting. The dipping intervals were selected as the periods when the source count rate clearly deviates from the linearly increasing trend 
Table 1

Spectral Fitting of Swift J1658.2-4242 in the Hard State

\begin{tabular}{|c|c|c|c|c|}
\hline Component & Parameter & Persistent (Model 3) & Persistent (Model 4) & Dipping (Model 5) \\
\hline TBNEW & $N_{\mathrm{H}}\left(\times 10^{23} \mathrm{~cm}^{-2}\right)$ & $1.85_{-0.10}^{+0.07}$ & $1.80 \pm 0.08$ & $\ldots$ \\
\hline \multirow[t]{2}{*}{$\mathrm{PARCOV} *(\mathrm{TBNEW} * \mathrm{CABS})$} & $N_{\mathrm{H}}\left(\times 10^{24} \mathrm{~cm}^{-2}\right)$ & $\cdots$ & $\cdots$ & $1.00_{-0.07}^{+0.08}$ \\
\hline & $f_{\text {cov }}(\%)$ & $\cdots$ & $\cdots$ & $34 \pm 1$ \\
\hline \multirow[t]{3}{*}{ XSTAR } & $N_{\mathrm{H}}\left(\times 10^{22} \mathrm{~cm}^{-2}\right)$ & $3.2_{-1.0}^{+1.4}$ & $2.0_{-1.0}^{+1.2}$ & $\ldots$ \\
\hline & $\log (\xi)\left(\log \left[\mathrm{erg} \mathrm{cm} \mathrm{s}^{-1}\right]\right)$ & $2.09_{-0.07}^{+0.17}$ & $2.00_{-0.08}^{+0.27}$ & $\cdots$ \\
\hline & $v_{\text {out }} / c$ & $0.077_{-0.007}^{+0.006}$ & $0.080_{-0.014}^{+0.007}$ & $\ldots$ \\
\hline \multirow[t]{9}{*}{ RELXILLLPCP } & $h\left(r_{\mathrm{g}}\right)$ & $<6.6$ & $<3.8$ & $\ldots$ \\
\hline & $a^{*}\left(c \mathrm{~J} / \mathrm{GM}^{2}\right)$ & $0.92_{-0.06}^{+0.04}$ & $>0.96$ & $\cdots$ \\
\hline & $i\left(^{\circ}\right)$ & $61_{-4}^{+3}$ & $64_{-3}^{+2}$ & $\ldots$ \\
\hline & $\Gamma$ & $1.69 \pm 0.02$ & $1.63 \pm 0.02$ & $\cdots$ \\
\hline & $\log (\xi)\left(\log \left[\mathrm{erg} \mathrm{cm} \mathrm{s}^{-1}\right]\right)$ & $3.49_{-0.08}^{+0.10}$ & $3.47_{-0.09}^{+0.19}$ & $\cdots$ \\
\hline & $A_{\mathrm{Fe}}($ solar $)$ & $0.60_{-0.09}^{+0.07}$ & $0.91_{-0.08}^{+0.54}$ & $\ldots$ \\
\hline & $k T_{\mathrm{e}}(\mathrm{keV})$ & $27 \pm 3$ & $22 \pm 1$ & $\ldots$ \\
\hline & $R_{\text {ref }}$ & 2.19 & 3.25 & $\cdots$ \\
\hline & Norm $\left(10^{-2}\right)$ & $1.5_{-0.7}^{+1.2}$ & $1.7_{-0.4}^{+0.1}$ & $\cdots$ \\
\hline \multirow[t]{3}{*}{ GAUSSIAN } & $E_{\mathrm{emi}}(\mathrm{keV})$ & $\cdots$ & $6.30 \pm 0.04$ & $\cdots$ \\
\hline & $\sigma(\mathrm{keV})$ & $\cdots$ & $0.23_{-0.06}^{+0.08}$ & $\ldots$ \\
\hline & Norm $\left(10^{-4}\right)$ & $\cdots$ & $5.7_{-1.1}^{+1.4}$ & $\ldots$ \\
\hline \multirow[t]{2}{*}{ XILLVERCP } & Norm $\left(10^{-2}\right)$ & $0.18 \pm 0.05$ & $\cdots$ & $\ldots$ \\
\hline & $\chi^{2} / \nu$ & $1650.0 / 1595$ & $1625.7 / 1593$ & $307.6 / 287$ \\
\hline
\end{tabular}

Note. There is no error estimation for the reflection fraction parameter, $R_{\text {ref }}$, as it is self-consistently calculated by the model in the lamppost geometry. In Model 5 , we add one extra absorption component, parcov* (TBnew*Cabs), to Model 4 in order to fit the dip spectra. The rest parameters are fixed at the best-fit value from Model 4.

(see Figure 2 for details). This results in an exposure time of $3.0 \mathrm{ks}$ and $3.3 \mathrm{ks}$ for FPMA and FPMB, respectively. To highlight the spectral difference during the dips, we plot the residuals of the dipping spectra when compared to the best-fit model for the persistent spectra (Model 4) in Figure 6(b). It is clear that dip spectra are harder, especially below $10 \mathrm{keV}$, suggesting that the spectral change is caused by absorption.

An increase in the neutral absorption column density and changes in photon-ionized absorbers are usually invoked to explain spectral change during dipping intervals (e.g., Díaz Trigo et al. 2006; Shidatsu et al. 2013). Evidence have also been found for progressive covering of the accretion disk corona as the dip progresses, during which the covering factor of the absorber was measured to increase, approaching the maximum value at the dip minimum (e.g., Smale et al. 2002; Church \& Bałucińska-Church 2004). Our best-fit model for the dip spectra adds one extra partial covering neutral absorber to the persistent spectral model (Model 4). During the dips, electron scattering by the absorber could reduce the Comptonized emission from the corona, producing a nearly energy-independent decrease in intensity (e.g., Parmar et al. 1986; Smale et al. 2002). Therefore, we also include a cabs model to account for the effects of absorbing material scattering X-ray photons away from the line of sight, and link the absorption column density in the cabs model with that of the partial covering absorber. The full model setup is TBnew* (partcov* (TBnew*Cabs) ) *XSTAR* (relxilllpCp+Gaussian) (Model 5). Corresponding parameters in Model 5 are fixed at the best-fit values from Model 4, with only two parameters left to vary freely. The dip spectra can be adequately modeled after including the extra absorption component $\left(\chi^{2} / \nu=307.6 / 287=1.07\right.$, with no obvious residuals in Figure 6(c)). The best fit requires a neutral absorber with the absorption column density of $N_{\mathrm{H}}=\left(1.00_{-0.07}^{+0.08}\right) \times 10^{24} \mathrm{~cm}^{-2}$ and

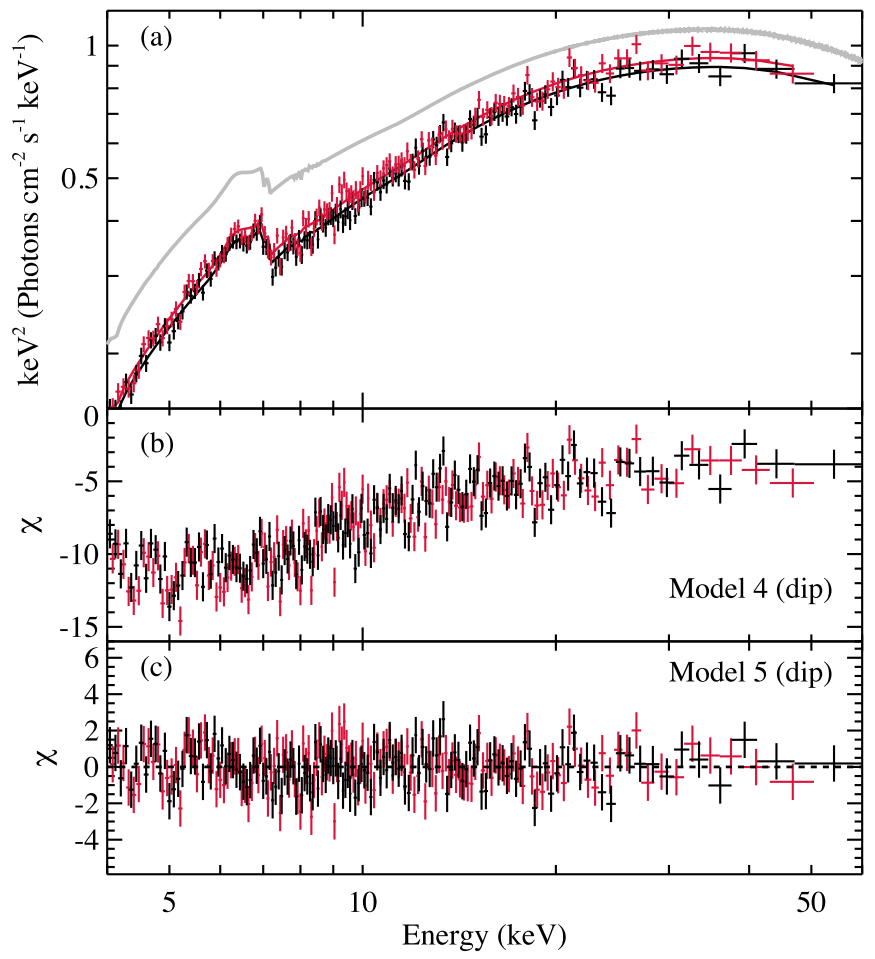

Figure 6. (a) Residuals of the dipping spectra compared to Model 4 (best model for the persistent spectra). (b) Residuals of the dipping spectra from the best-fit model (Model 5). The spectra have been rebinned for display clarity.

a partial covering factor of $f_{\text {cov }}=(34 \pm 1) \%$. The fit confirms that spectral changes during the dips can be fully accounted for by photoelectric absorption and Compton scattering from an additional neutral absorber. 

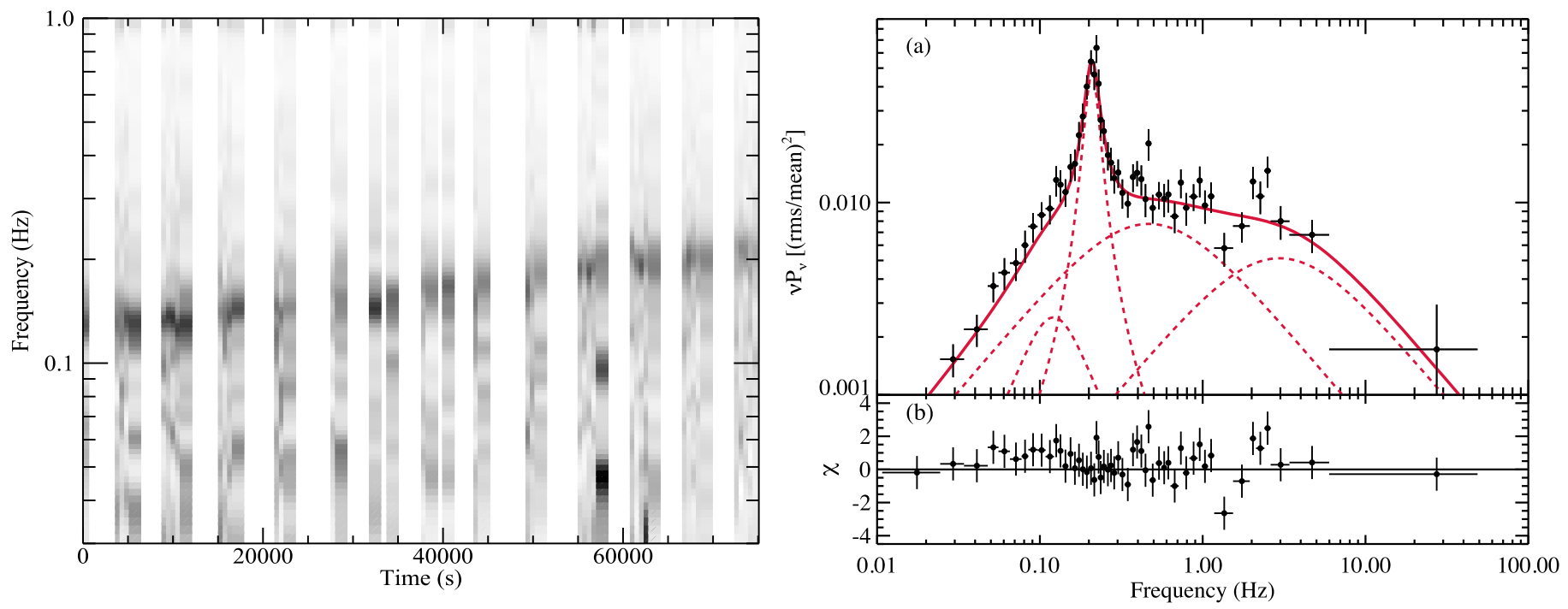

Figure 7. Left: dynamical power spectrum of Swift J1658.2-4242 from the NuSTAR observation. Low-frequency noise is present during the dipping period. Right: sample power spectrum extracted from the last two orbits fitted with a multi-Lorentizan model. The power spectrum here has been rebinned for display clarity.

\section{Low-frequency QPO}

For the NUSTAR timing analysis, we first applied a barycenter correction to the event files, transferring the photon arrival times to the barycenter of the solar system using JPL Planetary Ephemeris DE-200. The cross-power density spectra (CPDS) were generated using MaLTPyNT (Bachetti 2015) following the standard procedure. CPDS measures the signals in phase between the two NUSTAR modules (FPMA and FPMB), which helps to reduce dead time distortions to the power density spectra (Bachetti et al. 2015). We selected a light curve binning of $2^{-8} \mathrm{~s}$ and calculated the CPDS in $512 \mathrm{~s}$ intervals. The power spectra were generated using the rms normalization, and were geometrically rebinned by a factor of 1.03 to reach nearly equally spaced frequency bins in the logarithmic scale.

A low-frequency QPO is clearly detected by NuSTAR. The QPO frequency steadily increased from $\sim 0.14$ to $\sim 0.21 \mathrm{~Hz}$ during the NUSTAR exposure, as shown in the dynamical power spectrum (Figure 7, left panel). We extract an example CPDS from only the last two orbits, and fit it in XSPEC with a unity response file. We use a multi-Lorentzian model, which is commonly used to fit the power spectra of black hole binaries (e.g., Nowak 2000). Our model for the power density spectrum includes four Lorentz functions: two Lorentzians with the centroid frequency fixed at zero to fit for the underlying continuum, one Lorentzian for the QPO, and one for the possible subharmonic with the frequency linked with half the fundamental QPO frequency. The sample power spectrum can be adequately fitted with no obvious structure left in the residuals, $\chi^{2} / \nu=292.6 / 243=1.20$ (see Figure 7, right panel). The QPO peak averaged over the last two orbits can be well constrained to be at the frequency of $0.207_{-0.002}^{+0.003} \mathrm{~Hz}$, with a $Q$-value of $4.5 \pm 0.9$ and a fractional rms amplitude of $12.7 \pm 0.8 \%$. The low QPO frequency, the increasing trend of the centroid frequency with the rising source flux, and the underlying noise continuum in the power density spectrum are consistent with type-C QPOs during hard states of black hole binary outbursts (Wijnands \& van der Klis 1999; Casella et al. 2004, 2005).

\section{Discussion}

In this work, we performed an X-ray spectral and timing analysis of the recently discovered X-ray transient Swift J1658.2-4242, using data from joint NuSTAR and Swift observations. The shape of the broadband X-ray spectral continuum and the detection of a low-frequency QPO is similar to the properties of a black hole binary in the hard state. Given that no coherent pulsation or type I X-ray burst are detected, this suggests that the source is a strong black hole binary candidate. From spectral modeling, the highly absorbed nature of Swift J1658.2-4242 is confirmed, with the neutral absorption column density well measured as $N_{\mathrm{H}}=(1.80 \pm 0.08) \times$ $10^{23} \mathrm{~cm}^{-2}$. The hard photon index of $\Gamma=1.63 \pm 0.02$ and the simultaneous detection of a jet in the radio band (Russell et al. 2018) suggests that Swift J1658.2-4242 was in the hard state when the observations were taken. It was most likely in the bright phase of the hard state, as the source was reported to enter the hard-intermediate state four days later (Beri et al. 2018). The averaged non-dip flux is about $30 \mathrm{mCrab}$ in the $2-10 \mathrm{keV}$ band during the NuSTAR exposure, and the absorption-corrected source luminosity is $L_{\mathrm{x}, 0.1-500 \mathrm{keV}}=3.9 \times 10^{37} \times$ $(D / 8 \mathrm{kpc})^{2} \mathrm{erg} \mathrm{s}^{-1}$. Currently, the distance, $D$, to Swift $\mathrm{J} 1658.2-4242$ is unknown and the high absorption in the interstellar medium along the line of sight makes it difficult to identify the optical counterpart.

Strong disk reflection features are shown in the NUSTAR spectra. Modeling the reflection features with the selfconsistent disk reflection model relxilllpCp indicates that the central black hole is rapidly spinning with the spin parameter of $a^{*}>0.96$, and the inner disk is viewed at a high inclination angle of $i=64_{-3}^{+2 \circ}$. The inclination angle measured is consistent with that fact that absorption dips are detected in the light curves.

During spectral modeling of the reflection spectra in Section 5, we made the assumption that the inner accretion disk extends to the ISCO, which is not necessary true considering the possibility of a truncated disk in the hard state. The black hole spin and the inner disk radius are essentially degenerate parameters. The spin parameter is determined by measuring the location of the ISCO, which decreases 
monotonically from $9 r_{\mathrm{g}}$ for an extremely retrograde spinning black hole to $1.235 r_{\mathrm{g}}$ for a black hole with a maximum positive spin. Therefore, by assuming that the disk extends to the ISCO rather than being truncated, we would be conservatively fitting for a lower limit of the black hole spin. Likewise, a high black hole spin obtained this way rules out the possibility of significant disk truncation. If we fix the spin parameter at the maximum value of $a^{*}=0.998$ and instead fit for $R_{\text {in }}$, the bestfit value for the inner disk radius is $R_{\text {in }}=1.7_{-0.1}^{+0.2} r_{\mathrm{g}}$, with other parameters remaining at basically identical values. And solutions with $R_{\text {in }}>3.3 r_{\mathrm{g}}$ can be ruled out at a $5 \sigma$ confidence level.

A nearly maximal spin and the lack of significant disk truncation further support the hypothesis that Swift J1658.2-4242 contains a black hole primary. In the case of neutron stars, the Schwarzschild metric is normally a good approximation, and the spin parameter of neutron stars is expected to be less than 0.3 (e.g., Galloway et al. 2008). Also in neutron stars the disk must be truncated either at the surface of the star or at its magnetospheric radius, with the typical measured truncation radius as 6-15 $r_{\mathrm{g}}$ (e.g., Cackett et al. 2010; Miller et al. 2013; Ludlam et al. 2017), which significantly exceeds the constraint on the inner disk radius we obtained for Swift J1658.2-4242. The origin of QPOs in black hole binaries is still unclear despite being studied for decades. The lack of disk truncation plus the simultaneous detection of a type-C QPO in the hard state of a black hole binary candidate by $N U S T A R$ is in tension with the Lense-Thirring precession model for QPOs, one of the currently promising physical models to explain the origin of low-frequency QPOs, as in the LenseThirring precession model the inner accretion disk is generally required to be truncated (Stella \& Vietri 1998; Stella et al. 1999; Ingram et al. 2009).

An apparent absorption line is detected in the Fe $\mathrm{K}$ band centered at $7.03_{-0.03}^{+0.04} \mathrm{keV}$. If associated with ionized iron absorption, this implies the unusual presence of a disk wind in the hard state of a black hole binary. As only one absorption feature is clearly detected, the velocity shift and the ionization state of the absorber cannot be unambiguously determined, similar to the case of $4 \mathrm{U}$ 1630-472 discussed in King et al. (2014). The best fit favors a lower ionization state and a very high outflowing velocity, comparable to the extreme wind velocity reported in the black hole binary candidate IGR J17091-3624 (King et al. 2012). In the case of an extreme disk wind, the relatively narrow $\mathrm{Fe} \mathrm{K}$ emission line component required in the spectral modeling could be a part of a P-Cygni profile, arising from redshifted ionized iron line emission in the wind. However, we stress that an alternative solution of a higher ionization and moderate outflowing velocity cannot be ruled out from the spectral analysis, where the velocity shift of the ionized absorber is actually close to the absolute energy calibration uncertainty of NuSTAR. Swift J1658.2-4242 is an interesting source for studying the equatorial disk winds in binary systems. Future observations with high spectral resolution would be helpful to resolve this uncertainty.

We thank the referee for helpful comments that improved this work. D.J.W. acknowledges support from STFC Ernest Rutherford Fellowship. This work was supported under NASA contract No. NNG08FD60C and made use of data from the NUSTAR mission, a project led by the California Institute of Technology, managed by the Jet Propulsion Laboratory, and funded by the National Aeronautics and Space Administration. We thank the NUSTAR Operations, Software, and Calibration teams for support with the execution and analysis of these observations. This research has made use of the NuSTAR Data Analysis Software (NuSTARDAS), jointly developed by the ASI Science Data Center (ASDC, Italy) and the California Institute of Technology (USA).

\section{ORCID iDs}

Yanjun Xu (iD https://orcid.org/0000-0003-2443-3698 Fiona A. Harrison (iD https://orcid.org/0000-0003-2992-8024

Jamie A. Kennea (i) https://orcid.org/0000-0002-6745-4790

Dominic J. Walton (iD https://orcid.org/0000-0001-5819-3552 John A. Tomsick (iD https://orcid.org/0000-0001-5506-9855 Didier Barret (1) https://orcid.org/0000-0002-0393-9190 Andrew C. Fabian (iD https://orcid.org/0000-0002-9378-4072 Felix Fürst (ib) https://orcid.org/0000-0003-0388-0560 Poshak Gandhi (i) https://orcid.org/0000-0003-3105-2615 Javier A. García (D) https://orcid.org/0000-0003-3828-2448

\section{References}

Arnaud, K. A. 1996, in ASP Conf. Ser. 101, Astronomical Data Analysis Software and Systems V, ed. G. H. Jacoby \& J. Barnes (San Francisco, CA: ASP), 17

Bachetti, M. 2015, MaLTPyNT: Quick look timing analysis for NuSTAR data, Astrophysics Source Code Library, ascl:1502.021

Bachetti, M., Harrison, F. A., Cook, R., et al. 2015, ApJ, 800, 109

Beri, A., Belloni, T., Vincentelli, F., Gandhi, P., \& Altamirano, D. 2018, ATel, 11375,1

Burrows, D. N., Hill, J. E., Nousek, J. A., et al. 2005, SSRv, 120, 165

Cackett, E. M., Miller, J. M., Ballantyne, D. R., et al. 2010, ApJ, 720, 205

Casella, P., Belloni, T., Homan, J., \& Stella, L. 2004, A\&A, 426, 587

Casella, P., Belloni, T., \& Stella, L. 2005, ApJ, 629, 403

Church, M. J., \& Bałucińska-Church, M. 2004, MNRAS, 348, 955

Dauser, T., García, J., Parker, M. L., Fabian, A. C., \& Wilms, J. 2014, MNRAS, 444, L100

Díaz Trigo, M., Parmar, A. N., Boirin, L., et al. 2009, A\&A, 493, 145

Díaz Trigo, M., Parmar, A. N., Boirin, L., Méndez, M., \& Kaastra, J. S. 2006, A\&A, 445, 179

Done, C., Gierliński, M., \& Kubota, A. 2007, A\&ARv, 15, 1

El-Batal, A. M., Miller, J. M., Reynolds, M. T., et al. 2016, ApJL, 826, L12

Esin, A. A., McClintock, J. E., \& Narayan, R. 1997, ApJ, 489, 865

Fabian, A. C., Rees, M. J., Stella, L., \& White, N. E. 1989, MNRAS, 238, 729

Frank, J., King, A. R., \& Lasota, J. P. 1987, A\&A, 178, 137

Galloway, D. K., Muno, M. P., Hartman, J. M., Psaltis, D., \& Chakrabarty, D. 2008, ApJS, 179, 360

García, J., \& Kallman, T. R. 2010, ApJ, 718, 695

García, J. A., Dauser, T., Lohfink, A., et al. 2014, ApJ, 782, 76

Grebenev, S. A., Mereminskiy, I. A., Prosvetov, A. V., et al. 2018, ATel, 11306, 1

Grinberg, V., Eikmann, W., Kreykenbohm, I., \& Wilms, J. 2018, ATel, 11318,1

Harrison, F. A., Craig, W. W., Christensen, F. E., et al. 2013, ApJ, 770, 103 Ingram, A., Done, C., \& Fragile, P. C. 2009, MNRAS, 397, L101

Joinet, A., Kalemci, E., \& Senziani, F. 2008, ApJ, 679, 655

Kalberla, P. M. W., Burton, W. B., Hartmann, D., et al. 2005, A\&A, 440, 775 Kallman, T., \& Bautista, M. 2001, ApJS, 133, 221

King, A. L., Miller, J. M., Raymond, J., et al. 2012, ApJL, 746, L20 King, A. L., Walton, D. J., Miller, J. M., et al. 2014, ApJL, 784, L2

Krimm, H. A., Holland, S. T., Corbet, R. H. D., et al. 2013, ApJS, 209, 14 Kuulkers, E., Kouveliotou, C., Belloni, T., et al. 2013, A\&A, 552, A32 Kuulkers, E., Wijnands, R., Belloni, T., et al. 1998, ApJ, 494, 753 Lien, A. Y., Kennea, J. A., Barthelmy, S. D., et al. 2018, ATel, 11310, 1 Ludlam, R. M., Miller, J. M., Bachetti, M., et al. 2017, ApJ, 836, 140 Madsen, K. K., Harrison, F. A., Markwardt, C. B., et al. 2015, ApJS, 220, 8 Miller, J. M., Homan, J., Steeghs, D., et al. 2006, ApJ, 653, 525 Miller, J. M., Fabian, A. C., Kaastra, J., et al. 2015a, ApJ, 814, 87 Miller, J. M., Fabian, A. C., Wijnands, R., et al. 2002, ApJL, 570, L69 Miller, J. M., Parker, M. L., Fuerst, F., et al. 2013, ApJL, 775, L45 Miller, J. M., Raymond, J., Fabian, A. C., et al. 2012, ApJL, 759, L6 
Miller, J. M., Tomsick, J. A., Bachetti, M., et al. 2015b, ApJL, 799, L6

Motta, S., Belloni, T., \& Homan, J. 2009, MNRAS, 400, 1603

Neilsen, J., \& Lee, J. C. 2009, Natur, 458, 481

Nowak, M. A. 2000, MNRAS, 318, 361

Parmar, A. N., White, N. E., Giommi, P., \& Gottwald, M. 1986, ApJ, 308, 199

Ponti, G., Fender, R. P., Begelman, M. C., et al. 2012, MNRAS, 422, L11

Remillard, R. A., \& McClintock, J. E. 2006, ARA\&A, 44, 49

Russell, T. D., Miller-Jones, J. C. A., Sivakoff, G. R., \& Tetarenko, A. J. 2018, ATel, 11322, 1

Shidatsu, M., Ueda, Y., Nakahira, S., et al. 2013, ApJ, 779, 26

Smale, A. P., Church, M. J., \& Bałucińska-Church, M. 2002, ApJ, 581, 1286

Stella, L., \& Vietri, M. 1998, ApJL, 492, L59

Stella, L., Vietri, M., \& Morsink, S. M. 1999, ApJL, 524, L63
Tomsick, J. A., Lapshov, I., \& Kaaret, P. 1998, ApJ, 494, 747

Tomsick, J. A., Parker, M. L., García, J. A., et al. 2018, ApJ, 855, 3

Verner, D. A., Ferland, G. J., Korista, K. T., \& Yakovlev, D. G. 1996, ApJ, 465,487

Walton, D. J., Mooley, K., King, A. L., et al. 2017, ApJ, 839, 110

Walton, D. J., Tomsick, J. A., Madsen, K. K., et al. 2016, ApJ, 826, 87

White, N. E., \& Swank, J. H. 1982, ApJL, 253, L61

Wijnands, R., \& van der Klis, M. 1999, ApJ, 514, 939

Wilms, J., Allen, A., \& McCray, R. 2000, ApJ, 542, 914

Xu, Y., Harrison, F. A., García, J. A., et al. 2018b, ApJL, 852, L34

Xu, Y., Kennea, J. A., Harrison, F. A., \& Forster, K. 2018a, ATel, 11321, 1

Zdziarski, A. A., Johnson, W. N., \& Magdziarz, P. 1996, MNRAS, 283, 193

Życki, P. T., Done, C., \& Smith, D. A. 1999, MNRAS, 309, 561 